\title{
Calcul des débits des puits incomplets
}

\author{
PAR J. ZAOUI
}

INGÉNIEUR HYDRAULICIEN A LA SOGREAH, GRENOHLE

\begin{abstract}
Pour calculer, dans une nappe à surface libre, le débit d'un paits incomplet, avec écoulement à symétrie. axiale autour de l'ouvrage, on ne dispose que de formules semi-empiriques. Une comparaison de ces formules a montré que les meilleurs résultats d'ensemble étaient fournis par la formule de Tcharnyi. Si plasieurs puits s'influencent mutuellement, on propose une méthode itérative approchée qui a été appliquée à trois situations dans lesquelles les débits obtenus ont été très cohérents. Dans les cas praliques usuels, la méthode doit donner des résultats satisfaisants, surtout grâce à la possibilité d'effectuer simplement des essais comparatifs. En effet, un programme de calcul a la machine électronique a été écit : il permet de changer arbitrairement la répartition des puits et les paramètres de l'écoulement par l'introduction de quelques cartes perforées.
\end{abstract}

\section{INTRODUCTION}

Les nappes aquifères sont exploitées au moyen de puits ou de sondages qui peuvent présenter des caractéristiques diverses. En particulier, on peut distinguer deux types de puits :

- les puits complets, qui traversent la totalité de l'épaisseur d'une couche aquifère;

- les puits incomplets, qui ne traversent qu'une partie de cette couche (fig. 1).

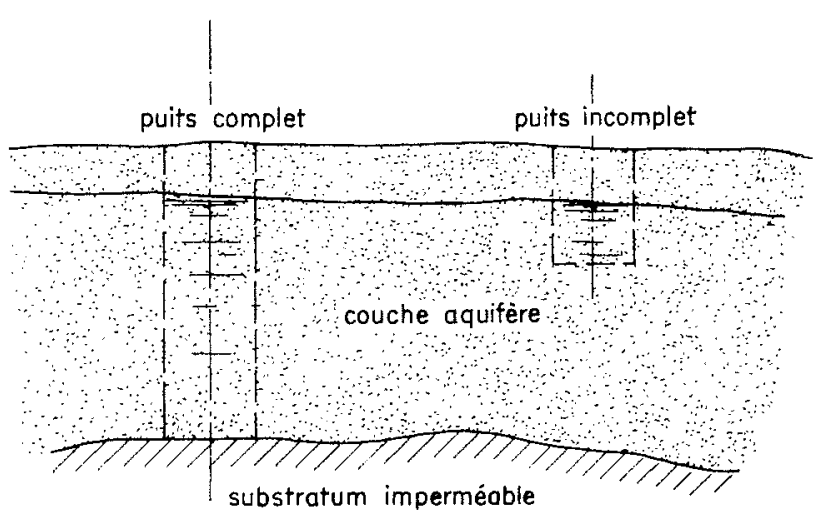

Fig. 1

Dans des cas relativement simples, il est possible de déterminer de manière rigoureuse la ou les relations qui existent entre les débits, les pressions ou les niveaux, et les caractéristiques des terrains aquifères, lorsqu'il s'agit de puits complets. Il n'en est pas de même lorsque les puits sont incomplets et l'objet de cette étude est de proposer des méthodes approximatives qui permettent de calculer les débits (ou les niveaux) d'un ou de plusieurs puits incomplets.

Ce problème présente un grand intérêt pratique, car la grosse majorité des puits existants ne sont pas creusés jusqu'au substratum. Cela tient, soit à la limitation des besoins que ces puits sont censés satisfaire, soit à l'imperfection des méthodes de fonçage, soit encore à l'éloignement excessif de l'assise.

S'il s'agit de projets de captage, ces méthodes sont également utiles; elles permettent, en particulier, de mieux définir les profondeurs optimales des ouvrages à construire.

\section{***}

\section{Hypothèses et limitations adoptées dans cette étude.}

-- Si l'on définit comme parfait un puits dont les parois n'opposent aucune résistance à la traversée du fluide, on ne considérera ici que des ouvrages parfaits. En pratique, la surface latérale et le fond du puits devront être largement crépinés pour que les pertes de charge singulières à travers le revêtement soient négligeables;

- On se limitera aux puits et sondages cylindriques, à génératrices verticales et à base circulaire, qui constituent la quasi-totalité des ouvrages existants;

- Il s'agira d'une nappe phréatique : Ja nappe admettra donc une surface libre où s'établira la pression atmosphérique (capillarité négligée); 
- La couche aquifère, homogène et isotrope, reposera sur un substratum horizontal et étanche;

- Un débit constant étant extrait de chacun des puits, on admettra que le régime permanent est établi et que la loi de Darcy s'applique en tout point du domaine.

\section{$*$}

Avec ces hypothèses, on examinera les trois cas suivants :

1. Ecoulement à symétrie de révolution autour d'un ouvrage unique;

2. Influence mutuelle des ouvrages;

3. Applications pratiques.

\section{I. - ECOULEMENT DE RÉVOLUTION}

La charge, en un point d'un écoulement, est donnée par Ia relation :

$$
\mathbf{H}=\frac{p}{\varpi}+z+\frac{\mathrm{V}^{2}}{2 g}
$$

dans laquelle :

H représente la charge en hauteur d'eau;

$p$ la pression;

๘ le poids spécifique du liquide;

$z$ la cote du point au-dessus d'un plan de référence;

$V$ la vitesse de l'écoulement, et

$g$ l'accélération due à la pesanteur.

Dans un écoulement en milieu poreux, l'énergie cinétique $V^{2} / 2 g$ est négligeable devant la somme des deux autres termes; donc :

$$
\mathrm{H}=\frac{p}{\bar{w}}+z
$$

$\mathrm{Si}$, en tous les points d'une nappe situés à une distance horizontale $\mathrm{R}$ de l'axe d'un puits, la charge $H$ est constante, l'écoulement présente une symétrie de révolution autour de ce puits.

\section{Formules de débit.}

Dans la situation théorique schématisée par la figure 2 , on ne connaît pas, à ce jour, de formule rigoureuse qui relie le débit extrait du puits aux autres caractéristiques de l'écoulement, mais de nombreux auteurs ont proposé des formules plus ou moins empiriques qu'ils ont établies de diverses manières: considérations théoriques, observations directes, modèles réduits, analogie électrique, etc.

Avec les notations représentées sur la figure 2, le tableau I rassemble les différentes formules que nous avons retenues,

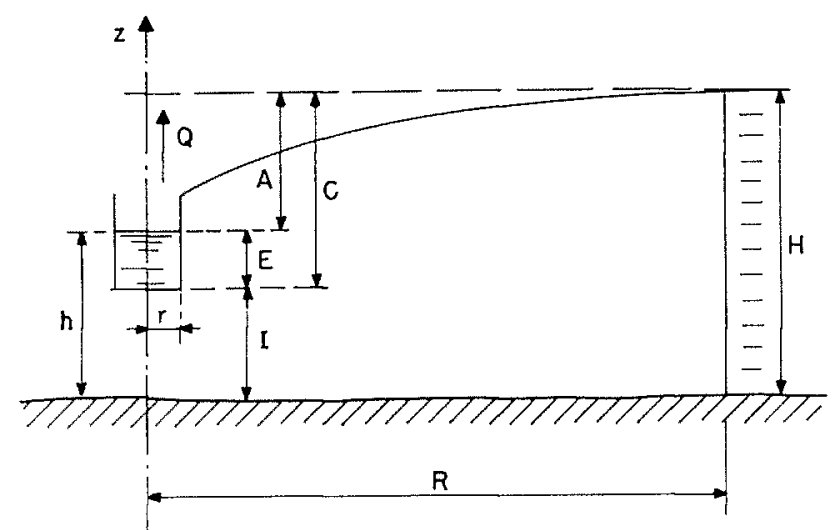

FIG. 2

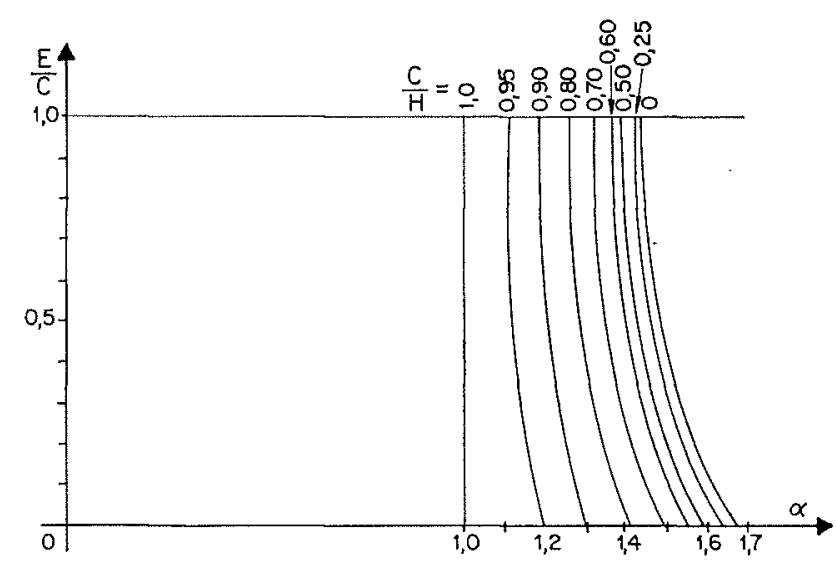

FIG. 3

Abaque de Breitenöder

\section{Comparaison des différentes formules.}

Dans nos recherches bibliographiques, nous avons trouvé les caractéristiques détaillées de 27 exemples concrets qui ont été traités :

-- 7 par la méthode de relaxation (Boreli),

- 2 par l'hydrodynamique graphique (Nahrgang),

- 18 par analogie électrique (Trofimenkov); ces caractéristiques ont été reportées sur le tableau II.

Pour pouvoir comparer les différentes formules, nous les avons appliquées à ces 27 exemples et nous avons obtenu les résultats, représentés en pourcentages des écarts, sur le tableau III.

On peut faire les remarques suivantes :

- l'écart absolu moyen, pour l'ensemble des formules, est voisin de $9 \%$. Ce pourcentage n'a rien d'excessif, si les formules sont appliquées à des ouvrages réels, à cause de l'ímprécision des mesures de perméabilité;

- les diverses formules donnent des écarts relativement importants pour les valeurs éle- 
Tableau I. - Formules semi-empiriques.

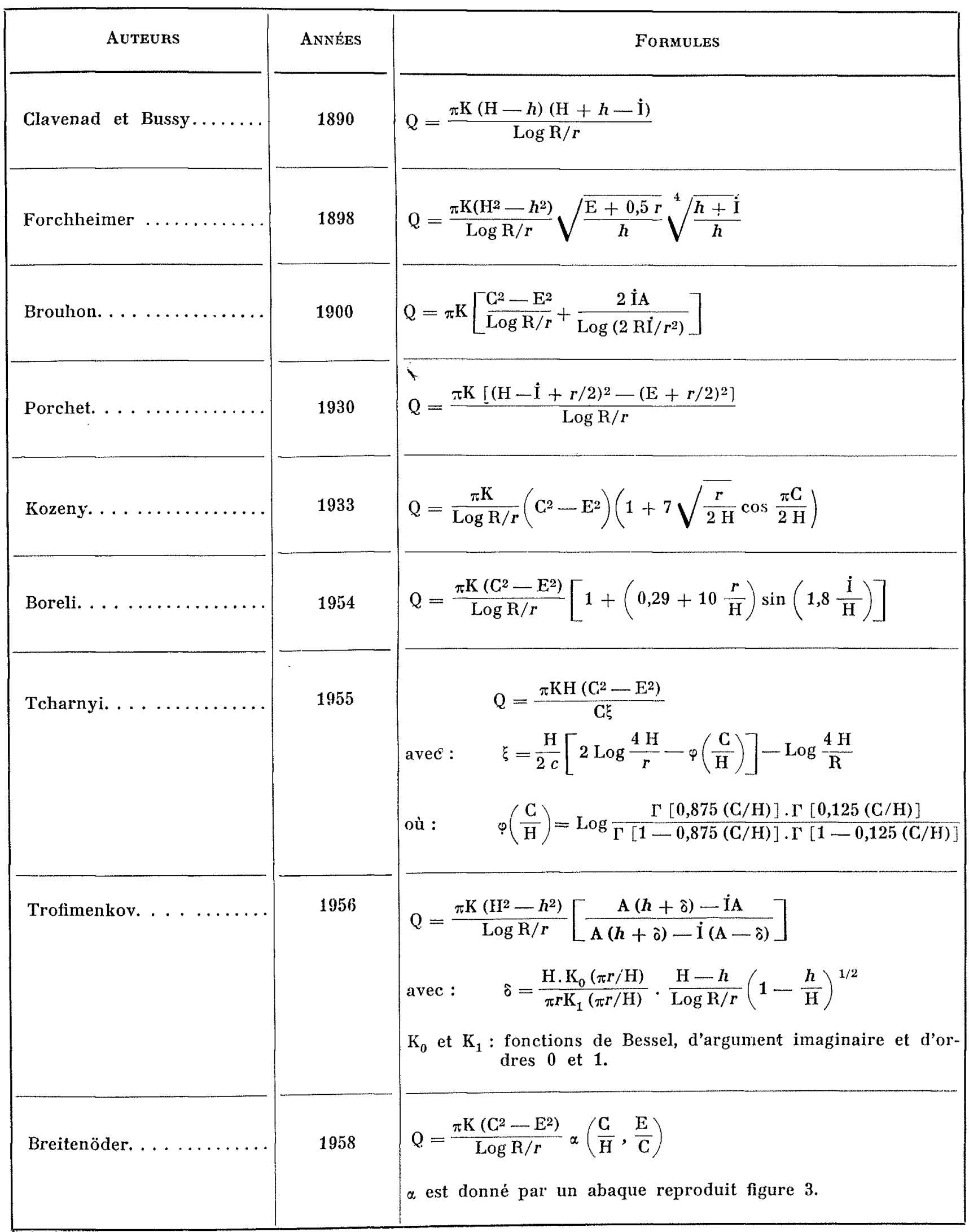


Tableau 11. - Caractéristiques des exemples concrets traités

\begin{tabular}{|c|c|c|c|c|c|c|}
\hline $\mathrm{N}^{\circ} \mathrm{CAs}$ & AUteur & $\bar{I} / \mathrm{H}$ & $\mathrm{E} / \mathrm{H}$ & $r / H$ & $\mathrm{R} / \mathrm{r}$ & $\mathrm{Q} / \pi \mathrm{KH}^{2}$ \\
\hline 1 & Boreli & 0,2650 & 0 & 0,0221 & 112 & 0,1451 \\
\hline 2 & $\triangleright$ & 0,2594 & 0,1730 & 0,0216 & 112 & 0,1344 \\
\hline 3 & 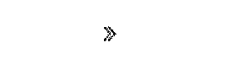 & 0,2618 & $0,3 \cdot 190$ & 0,0218 & 112 & 0,1115 \\
\hline 4 & $\gg$ & 0,2600 & 0,1733 & 0,0108 & 112 & 0,1296 \\
\hline .5 & $\gg$ & 0,2570 & 0,1713 & 0,0428 & 112 & 0,1468 \\
\hline 6 & $\gg$ & 0,5206 & 0,1935 & 0,0216 & 112 & 0,0589 \\
\hline 7 & $》$ & 0,0217 & 0,1739 & 0,0217 & 112 & 0,1997 \\
\hline 8 & Nalurgang & 0,500 & 0,298 & 0,0437 & 27,2 & 0,0695 \\
\hline 9 & $\gg$ & 0,500 & 0 & 0,0437 & 27,2 & 0,123 \\
\hline 10 & Trofimenkov & 0,2 & 0,6 & 0,0636 & 40,50 & 0,0871 \\
\hline 11 & $\$$ & 0,4 & 0,4 & 0,0636 & 40,50 & 0,0787 \\
\hline 12 & $»$ & 0,6 & 0,2 & 0,0636 & 40,50 & 0,0687 \\
\hline 13 & $>$ & 0,2 & 0,4 & 0,0636 & 40,50 & 0,1604 \\
\hline 14 & $》$ & 0,4 & 0,2 & 0,0636 & 40,50 & 0,1295 \\
\hline 15 & $\gg$ & 0.2 & 0,2 & 0,0636 & 40,50 & 0,2045 \\
\hline 16 & $\gg$ & 0,2 & 0,6 & 0,0467 & 54,55 & 0,0849 \\
\hline 17 & $\gg$ & 0,4 & 0,4 & 0,0467 & 54,55 & 0,0723 \\
\hline 18 & $\gg$ & 0,6 & 0,2 & 0,0467 & 54,55 & 0,0543 \\
\hline 19 & 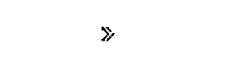 & 0,2 & 0,4 & 0,0467 & 54,55 & 0,1447 \\
\hline 20 & $\gg$ & 0,4 & 0,2 & 0,0467 & 54,55 & 0,1168 \\
\hline 21 & $\gg$ & 0,2 & 0,2 & 0,0467 & 54,55 & 0,1880 \\
\hline 22 & $\gg$ & 0,2 & 0,6 & 0,0275 & 90,90 & 0,0734 \\
\hline 23 & $\%$ & 0,4 & 0,4 & 0,0275 & 90,90 & 0,0643 \\
\hline 24 & $\gg$ & 0,6 & 0,2 & 0,0275 & 90,90 & 0,0475 \\
\hline 25 & $\gg$ & 0,2 & 0,4 & 0,0275 & 90,90 & 0,1295 \\
\hline 26 & $»$ & 0,4 & 0,2 & 0,0275 & 90,90 & 0,1052 \\
\hline 27 & $\gg$ & 0,2 & 0,2 & 0,0275 & 90,90 & 0,1614 \\
\hline
\end{tabular}


Tableau III. - Application des formules aux exemples connus (pourcentages d'écart)

\begin{tabular}{|c|c|c|c|c|c|c|c|c|c|c|}
\hline $\begin{array}{c}\mathrm{N}^{\circ} \\
\text { DU CAS }\end{array}$ & 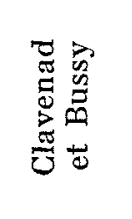 & 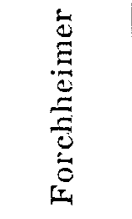 & 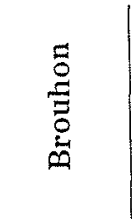 & $\begin{array}{l}\overrightarrow{\mathscr{g}} \\
\overline{0} \\
\dot{0} \\
\dot{0}\end{array}$ & 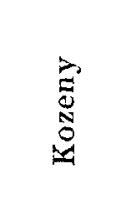 & $\begin{array}{l}\overline{0} \\
\vdots 0 \\
0\end{array}$ & 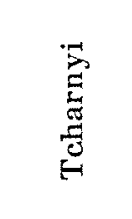 & 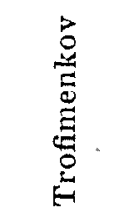 & 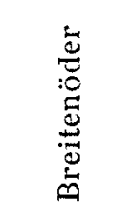 & \\
\hline 1 & $+9,5$ & $-67,0$ & $+13,0$ & $-19,7$ & $+5,7$ & - & $-5,6$ & $+\quad 2,0$ & $+16,0$ & 17,3 \\
\hline 2 & $+4,9$ & $-5,7$ & $+9,7$ & $-16,4$ & $+8,8$ & - & $--\quad 2,0$ & $+\quad 0,4$ & $+14,0$ & 6,2 \\
\hline 3 & 0 & $+2,0$ & $+\quad 4,0$ & $-17,5$ & $+\quad 7,2$ & - & $-3,5$ & $-1,3$ & $+\quad 7,0$ & 5,3 \\
\hline 4 & $+8,8$ & $-4,2$ & $+10,0$ & $-14,3$ & $+\quad 4,6$ & 一 & $--3,1$ & $+\quad 1,8$ & $+18,0$ & 8,1 \\
\hline 5 & $-3,3$ & $-10,9$ & $+\quad 3,2$ & $-21,0$ & $+9,7$ & - & $-4,8$ & $-4,0$ & $+\quad 5,5$ & 7,8 \\
\hline 6 & $+29,0$ & $+4,6$ & $+35,6$ & $-25,8$ & $+20,8$ & - & $-\quad 3,2$ & $+13,8$ & $+5,5$ & 17,3 \\
\hline 7 & 0 & $+1,7$ & $+1,0$ & 0 & $+0,3$ & - & 0 & 0 & $+3,0$ & 0,8 \\
\hline 8 & $+15,0$ & $+13,7$ & $+15,0$ & $-26,6$ & $+22,3$ & $+11,0$ & $-1,0$ & $+13,0$ & - & 14,7 \\
\hline 9 & $+23,6$ & $-54,0$ & $+22,8$ & $-33,4$ & $+\quad 6, \overline{5}$ & $-3,3$ & $-13,9$ & $-7,3$ & - & 20,6 \\
\hline 10 & $+\quad 0,5$ & $+\quad 6,7$ & $+11,4$ & $-8,8$ & $+21,2$ & $+16,1$ & $+12,4$ & - & $+9,6$ & 10,8 \\
\hline 11 & $-3,1$ & $+8,5$ & $+2,0$ & $-26,8$ & $+20,0$ & $+9,3$ & $+6,1$ & - & $-4,8$ & 10,1 \\
\hline 12 & $-4,9$ & $-11,4$ & $+0,3$ & $-47,5$ & $-5,1$ & $-16,0$ & $-12,5$ & - & $-32,0$ & 16,2 \\
\hline 13 & $-5,0$ & $-2,6$ & $-0,7$ & $-14,6$ & $+12,8$ & $+\quad 7,5$ & $+4,5$ & - & $+3,0$ & 6,4 \\
\hline 14 & $-5,8$ & $-5,0$ & $+\quad 7,0$ & $-27,8$ & $+16,6$ & $+\quad 6,0$ & $+2,9$ & - & $-\quad 3,9$ & 9,4 \\
\hline 15 & $-4,2$ & $-0,5$ & $+1,0$ & $-15,4$ & $+12,8$ & $+7,6$ & $+2,2$ & - & $+\quad 7,5$ & 6,4 \\
\hline 16 & $-5,1$ & $-0,1$ & $-1,8$ & $-14,6$ & $+9,8$ & $+5,8$ & $+4,4$ & - & $+\quad 3,3$ & 5,6 \\
\hline 17 & $-2,6$ & $+2,2$ & $+\quad 1,9$ & $-27,5$ & $+13,0$ & $+4,6$ & $+2,8$ & - & $-3,9$ & 7,3 \\
\hline 18 & $+11,3$ & $+1,8$ & $+16,8$ & $-40,1$ & $+3,3$ & $-\quad 7,2$ & $-3,5$ & - & $-19,9$ & 13,0 \\
\hline 19 & $-\quad 2,8$ & $-1,5$ & $+1,2$ & $-13,6$ & $+10,6$ & $+6,7$ & $+5,0$ & - & $+5,8$ & 5,9 \\
\hline 20 & $+\quad 3,2$ & $-4,5$ & $-8,9$ & $-27,4$ & $-11,9$ & $+3,4$ & $+1,4$ & - & $-1,7$ & 7,8 \\
\hline 21 & $-3,7$ & $-6,1$ & $+0,9$ & $--16,2$ & $+\quad 6,4$ & $-2,6$ & $+1,1$ & - & $+5,7$ & 5,3 \\
\hline 22 & $-3,4$ & $+1,1$ & $-0,3$ & $-13,5$ & $+5,5$ & $+9,0$ & $+4,8$ & - & $+5,3$ & 5,4 \\
\hline 23 & $\ldots 3,6$ & $+\quad 0,2$ & $+\quad 0,5$ & $-29,1$ & $+\quad 2,2$ & $-5,2$ & $-2,4$ & -- & $-5,2$ & 6,1 \\
\hline 24 & $+15,0$ & $+\quad 0,4$ & $+16,8$ & $-41,2$ & $-7,2$ & $-15,8$ & $-8,3$ & - & $-19,2$ & 15,5 \\
\hline 25 & $-4,4$ & $-4,1$ & $-1,2$ & $-15,8$ & $+2,6$ & $+\quad 6,0$ & $+1,5$ & - & $+4,2$ & 5,0 \\
\hline 26 & $+\quad 0,9$ & $--8,6$ & $+5,7$ & $-29,7$ & $\begin{array}{l}-0,2 \\
-\end{array}$ & $-7,2$ & $-4,6$ & - & $-3,7$ & 7,6 \\
\hline 27 & $-1,2$ & $-5,5$ & $+\quad 2,9$ & $-15,1$ & $+3,0$ & $+6,6$ & $+\quad 3,3$ & - & $+8,4$ & 5,8 \\
\hline $\begin{array}{l}\text { Ecart absolu } \\
\text { extrême }\end{array}$ & 29,0 & 67,0 & 35,6 & 47,5 & 22,3 & 16,1 & 13,9 & 13,8 & 32,0 & 67,0 \\
\hline $\begin{array}{c}\text { Ecart absolu } \\
\text { moyen }\end{array}$ & 6,5 & 8,7 & 7,2 & 22,2 & 9,3 & 7,8 & 4,5 & 4,8 & 8,7 & $\sim 9 \%$ \\
\hline
\end{tabular}




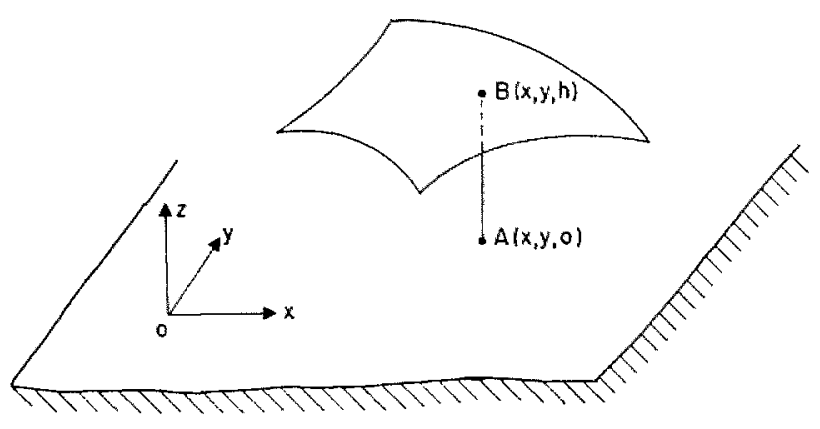

FIG. 4

vées du paramètre $\mathrm{I} / \mathrm{H}$. Au contraire, leur application au cas $n^{\circ} 7$, qui se caraclérise par une valeur très faible du rapport $\mathrm{I} / \mathrm{H}$, donne des résultats excellents;

- la formule de Porchet fournit des débits systématiquement trop faibles (plus de $22 \%$ en moyenne) : elle est donc à éviter;

- la formule de Forchheimer donne, dans les exemples 1 et 9 , des erreurs par défaut considérables. Or, ces deux cas se différencient des autres en ce que $\mathrm{E}$, hauteur d'eau dans le puits, est nulle. Cette formule ne devra pas être utilisée pour des hauteurs d'eau faibles;

- trois formules fournissent, dans tous les cas, des erreurs inférieures à $20 \%$ : ce sont celles de Boreli, Tcharnyi et Trofimenkov. Toutefois, l'écart absolu moyen de la formule de Boreli $(7,8 \%)$ est nettement plus élevé que celui des deux autres formules (respectivement 4,5 et $4,8 \%$ ).

En définitive, les deux formules les plus satisfaisantes sont celles de Trofimenkov et de Tcharnyi. Toutefois, nous préconiserons d'utiliser de préférence la formule de Tcharnyi, pour les raisons suivantes :

- la formule de 'Trofimenkov n'a pu être effectivement testée que dans les 9 premiers cas;

- si l'on se limitait à ces 9 cas, Ia formule de Tcharnyi donnerait un écart absolu moyen de $4,1 \%$ contre $4,8 \%$ à Trofimenkov;

- nous connaissons l'origine de l'établissement de la formule de Tcharnyi. Celui-ci s'est contenté de transposer, dans le cas d'un écoulement à surface libre, la formule établie théoriquement par Muskat dans le cas d'une nappe en charge. Bien que cette dernière formule ait été établie moyennant quelques hypothèses restrictives, ces hypothèses sont tout à fait raisonnables dans les applications pratiques usuelles.

La formule de Trofimenkov, ne comportant pas de constante numérique, a dû être établie à partir de considérations théoriques, mais nous ne les connaissons pas;
- il est évident que le débit $Q$ doit être borné supérieurement lorsque le substratum s'éloigne indéfiniment, toutes choses égales par ailleurs. Or, cela est vrai pour la formule de Tcharnyi, mais non pour celle de Trofimenkov.

La conclusion de ce premier chapitre semble s'imposer :

A moins de tomber, par hasard, au voisinage d'un des 27 cas qui ont été passés en revue, il paraît préférable, avec les éléments que nous avons rassemblés, d'utiliser la formule de Tcharnyi pour le calcul du débit d'un puits incomplet dans un écoulement à symétrie axiale.

\section{II. - INFLUENCE MUTUELLE DES PUITS}

\section{Fonction potentielle.}

On choisit un trièdre de référence trirectangle oxyz tel que le plan xoy soit confondu avec le substratum étanche et l'on considère un segment vertical $\mathrm{AB}$ qui traverse toute l'épaisseur de la nappe (fig. 4). Le long de ce segment éloigné de toutes singularités, la pression exprimée en hauteur d'eau, possède une valeur moyenne $p_{m}$.

La relation : $\mathrm{I}(x, y)=h \cdot p_{m}$ définit une fonction I que l'on démontre être harmonique et que l'on prendra comme fonction potentielle.

\section{Hypothèse fondamentale.}

Si le domaine étudié comprend plusieurs puits $\mathrm{P}_{i}$, de rayon $r_{i}$, on admet que ces rayons sont négligeables vis-à-vis des autres dimensions horizontales qui interviennent dans le système.

\section{Principe du calcul.}

Dans ces conditions, un puits $\mathrm{P}_{i}$, de débit $Q_{i}$, isolé dans une nappe horizontalement indéfinie, crée en un point $\mathrm{M}_{j}$, situé à une distance $\rho_{i j}$ un potentiel élémentaire :

$$
\mathrm{I}_{i j}(x, y)=-\frac{\mathrm{Q}_{i}}{2 \pi \mathrm{K}} \cdot \log \mathrm{P}_{i j}+\text { Cte }
$$

Ce potentiel ne dépend pas de la façon dont les débits pénètrent dans les ouvrages correspondants; il est le même, que le puits soit complet ou incomplet. D'après la propriété bien connue de superposition des potentiels, le potentiel $\mathrm{I}_{j}$ au point $\mathbf{M}_{j}$, créé par l'ensemble des puits $\mathbf{P}_{i}$ a pour valeurs :

$$
\mathrm{I}_{j}(x, y)=\frac{1}{2 \pi \mathrm{K}} \sum_{\mathrm{i}} \mathrm{Q}_{i} \cdot \log \rho_{i i}+\mathrm{Cte}
$$


Dans la pratique, la nappe sera limitée et pourra comprendre d'autres singularités: la relation précédente reste valable à condition de remplacer la constante par une fonction $\varphi(x, y)$, qui peut d'ailleurs dépendre des $Q_{i}$ et $\rho_{i j}$, mais que l'on suppose connue pour l'instant.

Supposons que les débits $\mathrm{Q}_{i}$ soient donnés, la relation (3) permet de calculer le potentiel $\mathrm{I}(\boldsymbol{x}, \boldsymbol{y})$ en tous les points du domaine. Choisissons autour de chaque puits $\mathrm{P}_{i}$, un cylindre coaxial $\mathrm{C}_{i}$, admettant pour section droite la circonférence $\Gamma_{i}$, de rayon $R_{i}$, et ne renfermant pas d'autres singularités que $\mathrm{P}_{i}$. Désignons par $\mathrm{I}_{i}$, la valeur moyenne prise par la fonction potentielle le long de $\Gamma_{i}$ et définissons une charge hydraulique $\mathrm{H}_{i}$ par la relation :

$$
\mathrm{H}_{i}=\sqrt{2 \overline{\mathrm{I}}_{i}}
$$

On admet qu'en remplaçant les charges réelles existant sur le cylindre $\mathrm{C}_{i}$ par la charge constante fictive $\mathrm{H}_{i}$, on ne change pas le débit $\mathrm{Q}_{i}$ : cette propriété est vraie pour un puits complet; pour un puits incomplet, on le vérifiera a posteriori. On obtient alors à l'intérieur de $\mathrm{C}_{i}$ un écoulement à symétrie axiale auquel il est possible d'appliquer la formule de Tcharnyi. Certes, il s'agit du problème inverse de détermination du niveau dans le puits à partir du débit, mais il n'y a plus de difficultés théoriques et nous n'insisterons pas sur ce cas.

Supposons, au contraire, que les niveaux dans les puits soient donnés; on prendra alors un jeu de débits $Q_{i 1}$, arbitrairement choisis, par exemple $\mathbf{Q}_{i 1}=\mathbf{0}$. Comme nous venons de le voir, il est possible de définir des charges hydrauliques $\mathbf{H}_{i 1}$; donc par application de la formule de Tcharnyi, par exemple, on obtiendra des débits $Q_{i 2}$ et ainsi de suite. On dispose ainsi d'une méthode par approximations successives dont nous avons vérifié la convergence.

Il faut s'assurer de plus que les approximations consenties ne sont pas de nature à donner des résultats aberrants. En particulier, la méthode ne peut être valable qu'à la condition que les débits obtenus soient pratiquement indépendants des rayons $\mathrm{R}_{i}$, qui ont été introduits par nécessité de calcul, mais qui n'ont aucun sens physique.

\section{III. - APPLICATIONS PRATIQUES}

\section{Remarques préliminaires.}

1. Le calcul, par approximations successives, des débits d'un groupe de puits complets ou incomplets conduit à des masses de calculs consi- dérables pour peu que le nombre de puits s'influençant mutuellement dépasse quelques unités. Si la méthode est difficilement utilisable à l'aide d'une machine de bureau, elle se révèle particulièrement bien adaptée aux possibilités des calculateurs électroniques modernes. En effet, nous avons écrit un programme général de calcul électronique qui peut prendre en compte un nombre variable de puits dont les caractéristiques peuvent être quelconques.

2. Nous n'avons pas examiné le problème de l'injection dans un puits incomplet; donc, les puits de ce type devront forcément fournir un certain débit; par contre, il est possible d'introduire des débits dans des puits complets, car les formules restent valables (le débit change simplement de signe).

\section{Situation étudiée.}

Nous nous sommes limité, dans les applications pratiques que nous avons traitées, à une situation qui permette le calcul explicite de la fonction $\varphi(x, y)$. Le schéma correspondant est représenté sur les figures 5 et 6 ; il s'agit d'une bande perméable indéfinie comprise entre deux limites quasi-parallèles : un cours d'eau ou un

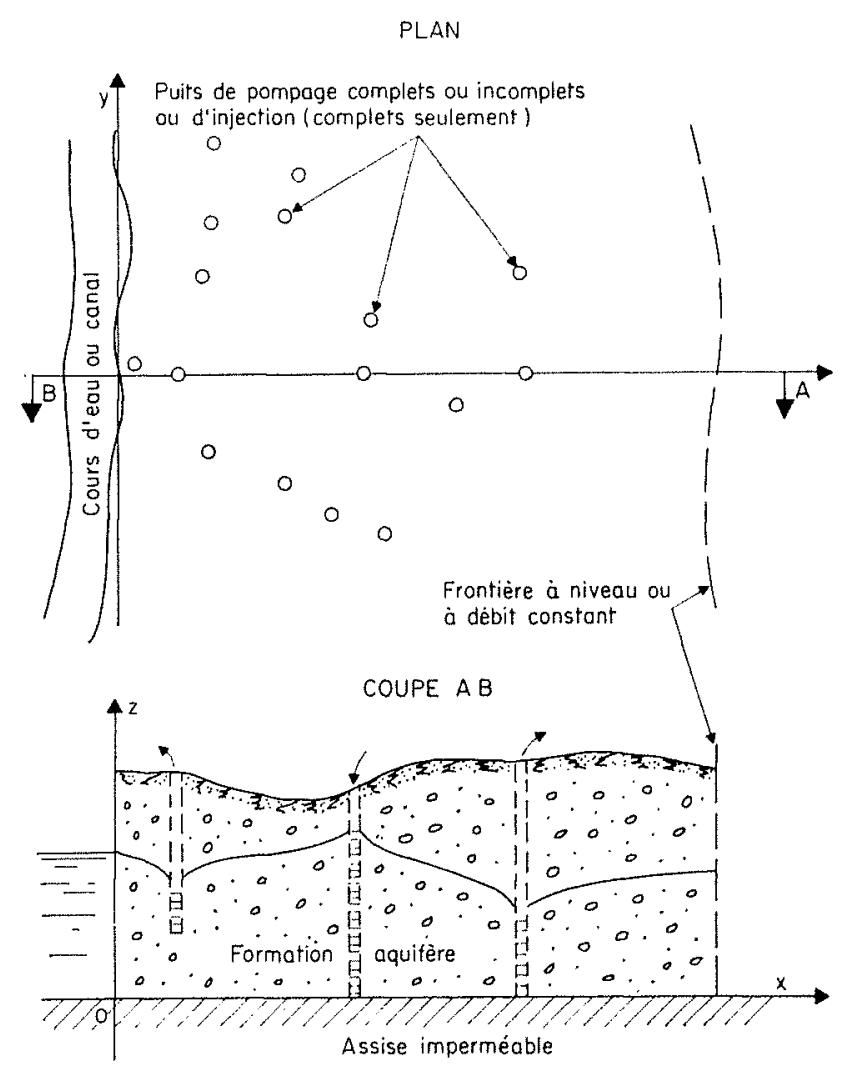

FIC. 5 et 6 
canal à niveau constant et une frontière à niveau ou à débit constant (cette frontière peut être reportée à l'infini).

\section{Premier exemple.}

On a pris un groupe de 4 puits disposés comme l'indique la figure 7 . En gardant invariables toutes les autres données, on a donné successivement aux rayons $R_{i}$ les valeurs suivantes : $5,20,35$ et $60 \mathrm{~m}$.
Pour chacun des puits, les écarts des 4 débits obtenus par rapport à la moyenne des débits extrêmes étaient inférieurs à $1 \%$, avec la formule de Tcharnyi.

A titre de vérification, on a effectué le même calcul à l'aide de la formule de Trofimenkov: pour chacun des puits $\mathrm{P}_{1}, \mathrm{P}_{3}$ et $\mathrm{P}_{4}$, les 4 débits obtenus étaient égaux à $3 \%$ près, mais pour le puits $P_{3}$, les écarts ont atteint $14 \%$. Ce résultat justifie à nouveau la préférence accordée à la formule de Tcharnyi.

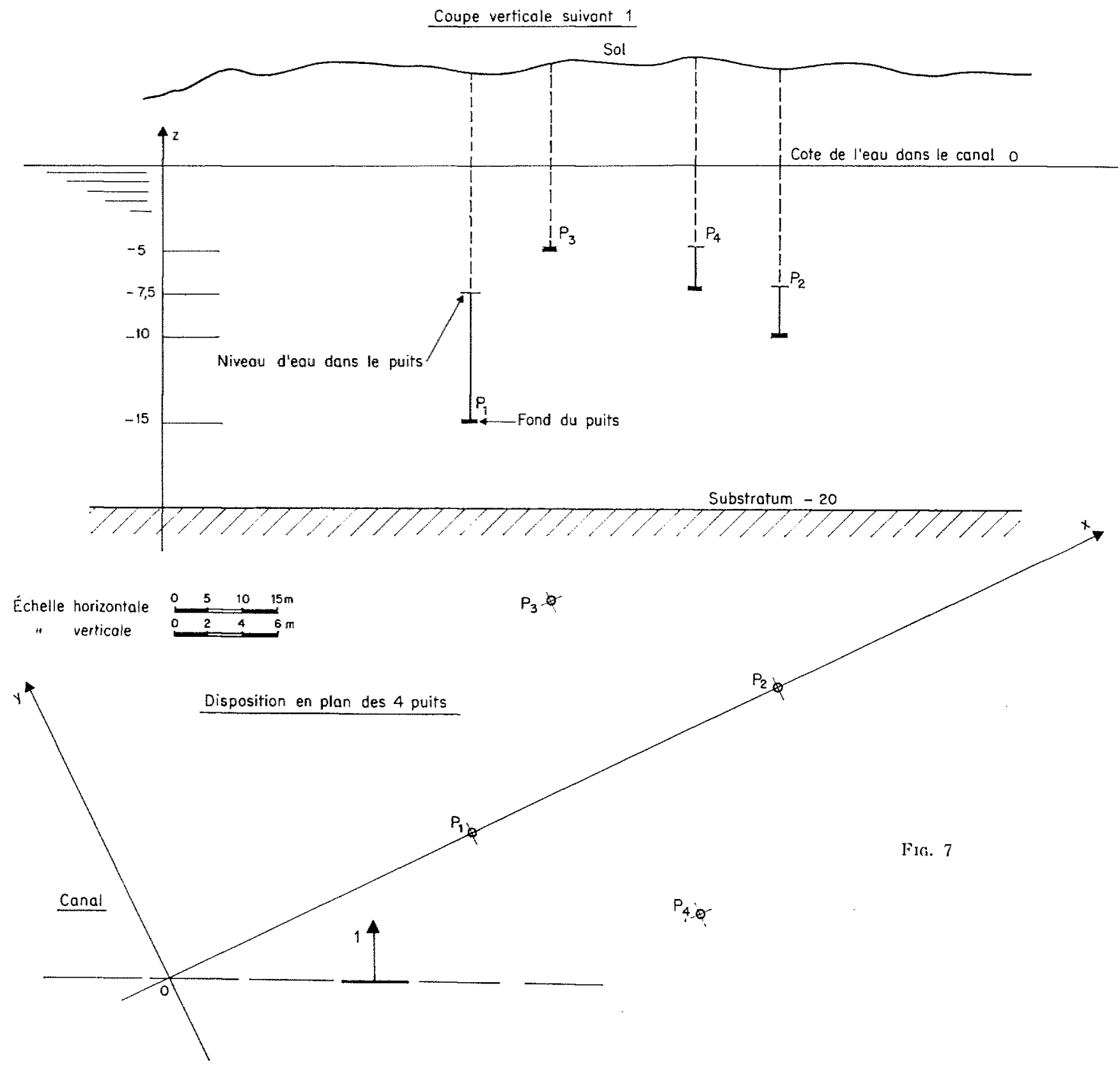



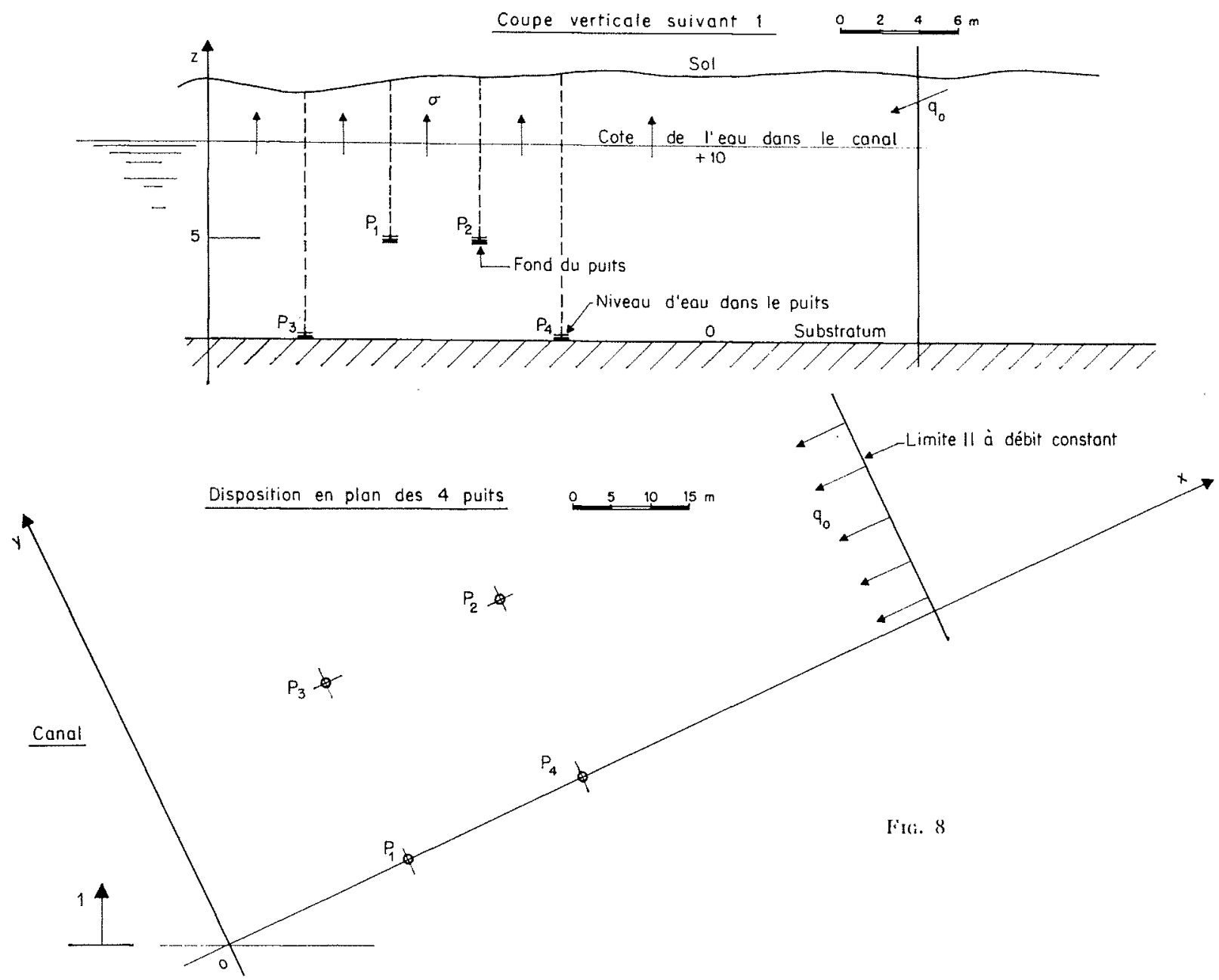

\section{Deuxième exemple.}

Le groupement comportait quatre puits, disposés comme l'indique la figure 8 , et dans lesquels le rabattement était total. On avait admis un apport linéaire constant $q_{0}$ par la frontière et en outre, on a supposé des pertes par évaporation uniformes (on démontre aisément que la méthode reste valable dans ce cas).

On a obtenu les résultats suivants qui se passent de commentaires :

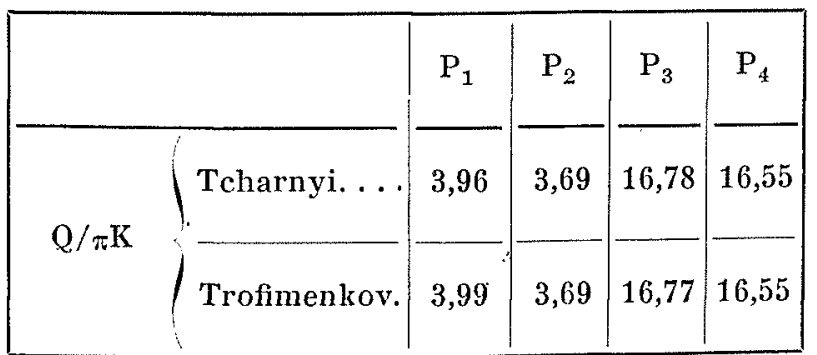

\section{Troisième exemple.}

Ce dernier exemple (fig. 9) était relatif à une batterie de 14 puits dont 7 étaient incomplets. Pour ceux-ci, la formule de Trofimenkov a donné des débits supérieurs à ceux fournis par la formule de Tcharnyi, mais les écarts sont minimes (de $2 \dot{a} 4 \%$ au maximum). Pour les puits complets, c'est évidemment l'inverse, mais les écarts sont encore plus faibles $(0$ à $1,3 \%)$.

\section{CONCLUSION}

Les exemples qui précèdent ne constituent pas une preuve irréfutable de la validité de la méthode proposée dans tous les cas possibles et imaginables. Dans les applications éventuelles, il est recommandé de procéder à des essais comparatifs en donnant plusieurs valeurs aux rayons $R_{i}$ et en utilisant successivement, si possible, les formules de Tcharnyi et de Trofimen- 


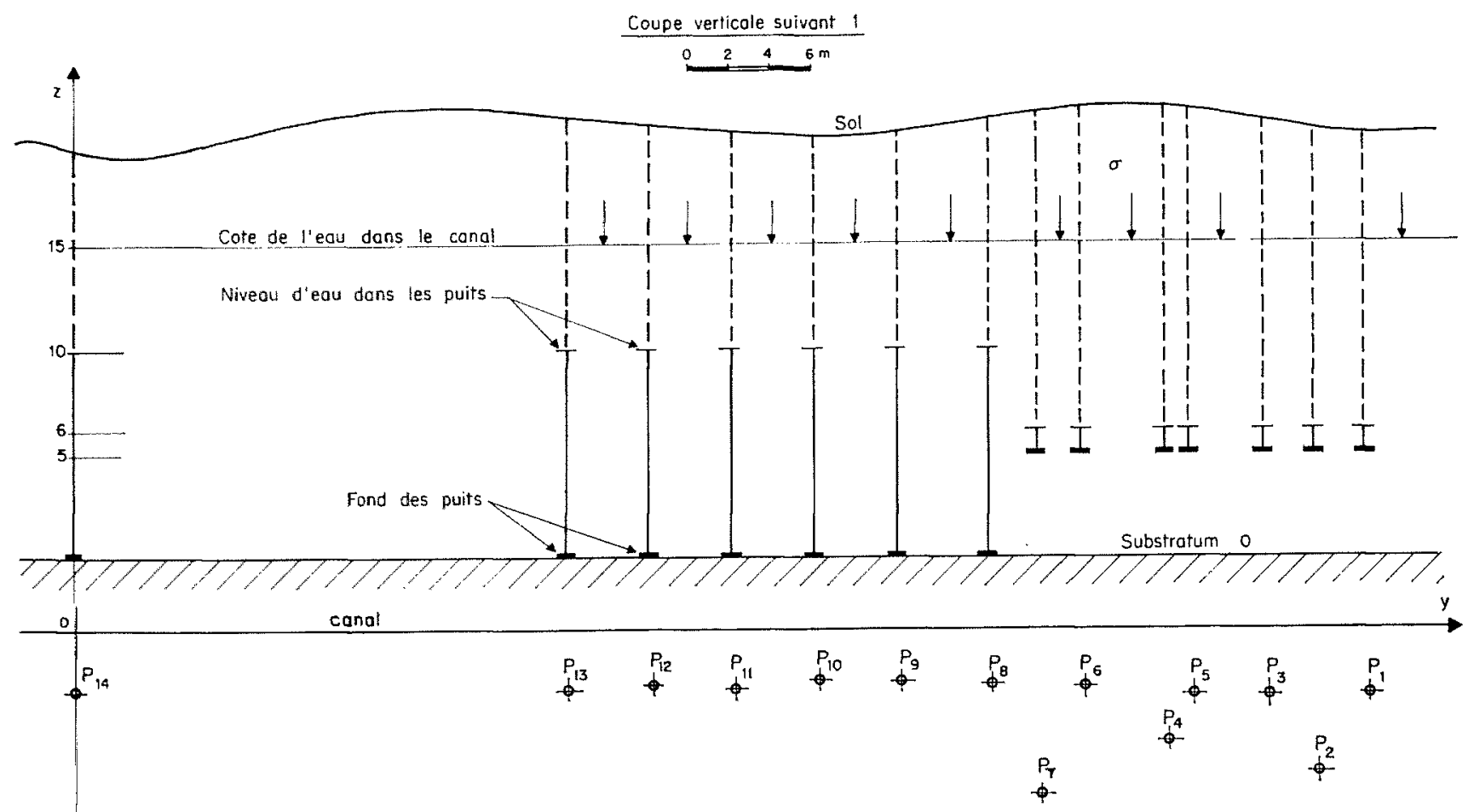

Disposition en plan des 14 puits

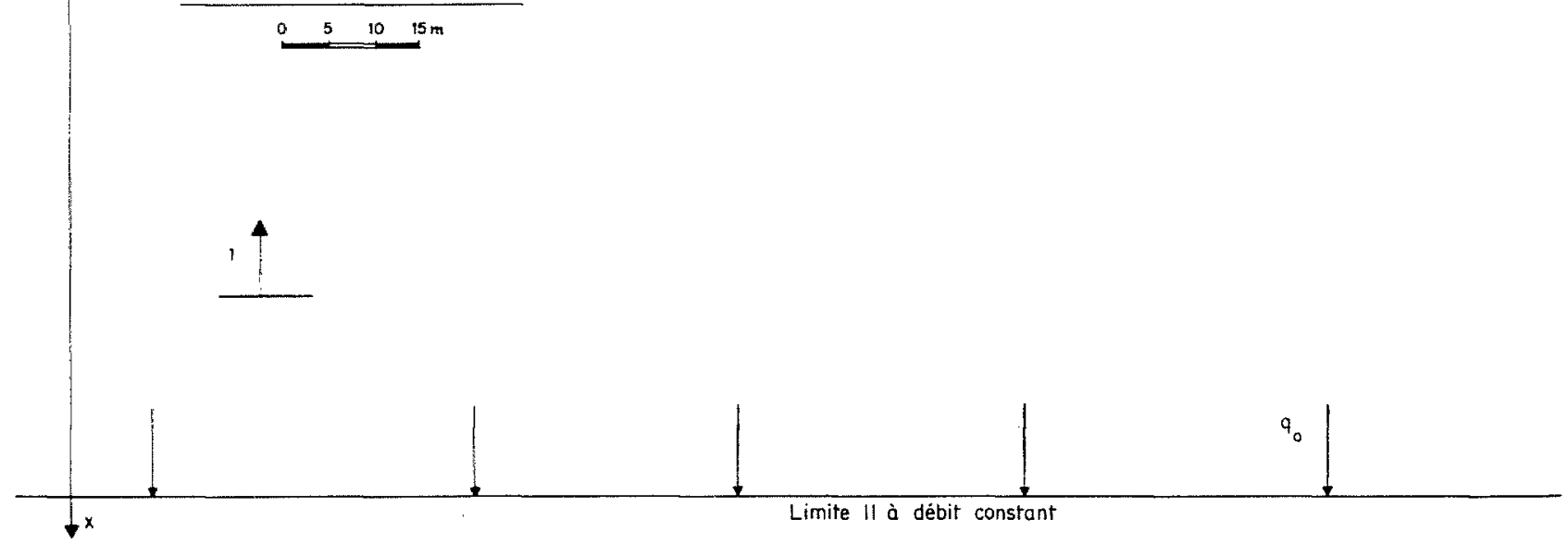

Fig. 9

kov. D'ailleurs, il serait très simple d'employer une relation établie ou future qui se révèlerait plus satisfaisante.

D'autre part, la situation que nous avons choisie pour éprouver la méthode de calcul a l'avantage de la simplicité, mais elle peut être remplacée par des schémas plus complexes, pourvu que la détermination de la fonction $\varphi(x, y)$ soit possible.

Enfin, les résultats obtenus montrent que les hypothèses adoptées sont raisonnables et que la méthode est satisfaisante dans les cas pratiques usuels; il ne faut pas oublier qu'un terrain naturel n'est jamais parfaitement homogène et que les mesures de perméabilité ne sont pas très précises. Il faut évidemment disposer d'un calculateur électronique et écrire le programme de calcul correspondant, mais en contrepartie, l'application répétée de ce programme à des répartitions de puits tout à fait arbitraires ne demande que la préparation de quelques cartes perforées.

\section{BIBLIOGRAPHIE}

Bonelr : Contribution à l'étude des milieux poreux. Thèse présentée à la Faculté des Sciences de Grenoble, janvier 1954 .

Nahrgang: Zur Theorie des vollkommenen und unvollkommenen Brunnens. 
Trofmenkov : Calcul des puits incomplets dans le cas d'une filtration sans charge. Construction Hydrotechnique, Moscou, $\mathrm{n}^{\circ} 11,1956$.

Poncher : Congrès de l'eau en Grau, 1930.

Forch hermer : Hydraulik. Berlin, 1930.

Tcharnyi : Pompage simultané de pétrole et d'eau sousjacente dans un puits incomplet. $C$. $R$. de l'Académie des Sciences de l'U.R.S.S.; $\mathrm{n}^{\circ} 2,1955$.

Clavenad et Bussy : Mémoire sur la filtration. Ann. des Ponts et Chaussées, 1890.
Brouhon : Cité dans : Grandottr. Compendio pratico di idrologia sottoranea. Gênes, 1956.

Kozeny : Theorie und Berechnung der Brunnen. Wasserkraft und Wasserwirtschaft, janvier 1933.

BreItenöDer : Der Wasseraudrang beim vollkommenen und undvollkommenen Brunnen mit freier Grundwasseroberfiache. Oesterreichische Wasserwirtschaft Vienne, mars 1958.

Muskat : The flow of homogeneous fluids through porous media. Ann. Harbor, 1946.

\title{
Calculating rates of flow from partially penetrating wells
}

\author{
BY J. ZAOUI
}

\begin{abstract}
A well or borehole which does not run through the entire depth of an aquifer is referred to as a partly penetrating well. As this type of well is more frequently encountered in practice than fully penetrating wells running through the entire formation from top to bottom, a method for calculating its discharge is obviously of interest.

Efforts have failed so far to establish from the usual assumptions - e.g. homogeneity, isotropy, steady flow, etc. - an analytical formula capable of giving strictly accurate discharge values for a single cylindrical well in a perfectly filtering unconfined aquifer with a symmetrical flow pattern about the well axis. A number of more or less empirical formulae are available, also results obtained from several concrete examples (the author knows of 27) which have been calculated by electrical analogy, relaxation and various other methods.

On comparing the discharge values yielded by the various formulae for the 27 cases know to the author with actual calculated values, it is seen that the best overall results are obtainable from Tcharnyi's formula, which also has the advantage of being derived from Muskat's formula for which the theoretical basis is known.

An approximate method can be used in the case of several interfering wells to calculate rates of flow in terms of water levels, or vice-versa, providing the well radii are small compared to the horizontal system dimensions. In this method, which is founded on potential theory, a coaxial zone is isolated around each well, in which the discharge is assumed to remain constant if the actual flow is replaced by an axisymmetrical flow with equivalent boundary conditions.

Well discharges are determined in terms of water level variations by a successive approximation procedure starting out from arbitrarily chosen discharge values.

The method was applied to conditions from which rates of flow could be calculated. Two separate groups of four wells were considered first, then one group of fourteen. A cross-check of the results obtained showed that the method satisfactory at least for the usual practical cases. It could no doubt be improved upon if a more general formula than Tcharnyi's were to be established.

Though the considerable mass of calculations in this method requires the use of an electronic computer, only a small number of punch cards need preparing in order to apply the programme repeatedly to any number of arbitrarily chosen well distributions.
\end{abstract}


